\title{
Preliminary studies: differences in microRNA expression in asthma and chronic obstructive pulmonary disease
}

\author{
Małgorzata Pietrusińska1 ${ }^{1}$ Aneta Pająk ${ }^{1}$, Paweł Górski' ${ }^{1}$ Piotr Kuna², Janusz Szemraj ${ }^{3}$, \\ Agnieszka Goździńska-Nielepkowicz ${ }^{4}$, Tadeusz Pietras ${ }^{5}$
}

${ }^{1}$ Department of Pneumology and Allergology, Institute of Onternal Medicine, Medical University of Lodz, Lodz, Poland ${ }^{2}$ Department of Internal Medicine, Asthma and Allergy, Medical University of Lodz, Lodz, Poland ${ }^{3}$ Department of Medical Biochemistry, Medical University of Lodz, Lodz, Poland ${ }^{4}$ Department of General Pulmonology and Oncology, Medical University of Lodz, Lodz, Poland ${ }^{5}$ Department of Clinical Pharmacology, Medical University of Lodz, Lodz, Poland

Adv Dermatol Allergol 2016; XXXIII (4): 276-280 DOI: $10.5114 / a d a .2016 .61603$

\begin{abstract}
Introduction: The asthma- and chronic obstructive pulmonary disease (COPD)-related morbidity has been increasing during the recent years. Both asthma and COPD are diseases of inflammatory etiology. The increasing interest in the pathomechanisms involved in the development of obstructive pulmonary diseases seems to be fully justified. Recent research has attempted to determine the associations of microRNA with the pathogenesis of pulmonary diseases. Aim: To assess the expression of microRNA in the blood sera of patients diagnosed with bronchial asthma and chronic obstructive pulmonary disease in comparison with healthy subjects.

Material and methods: In our study, at the preliminary stage, we compared the expression of miRNA in the groups of patients with asthma and COPD versus the control group of healthy subjects.

Results: A significant difference in hsa-miRNA-224, hsa-miRNA-339-5p, hsa-miRNA-382 in patients with asthma and COPD as compared with the controls was noted.

Conclusions: With such difference of expression of specific micro-RNA in serum of patient with asthma and COPD, those small non-coding RNA has to play a significant role in those diseases pathway. Therefore we expect to increase the size and differentation of the study groups in next studies.
\end{abstract}

Key words: microRNA, asthma, chronic obstructive pulmonary disease, serum.

\section{Introduction}

Bronchial asthma and chronic obstructive pulmonary disease (COPD) are very common respiratory tract diseases, which develop secondary to underlying inflammatory conditions of the airways. They lead to disorders of dyspnea type, reduced tolerance to exertion, wheezing. Despite the similarities of both diseases, they differ in the mechanisms of pathogenesis, changes in the airway structure, and reversibility of obstruction. A question arises whether or not microRNA plays a role in the pathogenesis of both diseases.

\section{MicroRNA}

microRNAs are non-coding DNA fragments comprising 21 to 23 nucleotides, involved in the process of transcrip- tion. The production of microRNA in mammals (studies carried out on mice) commences at the phase of RNA transcription by polymerase II, then, as pri-miRNA (several hundred to several thousand nucleotides) is exposed to Drosha endonuclease from DGCR8 - to form pre-miRNA (approximately 70-90 nucleotides), which is transported to the cytoplasm by exportin 5. It is exposed there to Dicer endonuclease. In this way, a double 22-nucleotide strand of hairpin structure is formed. The two strands are then separated. One of them subsequently forms, with Ago2 protein involved, the RNA-induced silencing complex (RISC). In the RISC form, miRNA may induce degradation or inhibit translation of mRNA then [1-3].

The functions of miRNA include primarily the regulation of gene expression at the posttranscriptional lev-

Address for correspondence: Małgorzata Pietrusińska, Clinical Department of Pulmonology and Allergology, University Hospital No. 1, Medical University of Lodz, 22 Kopcińskiego St, 91-498 Lodz, Poland, phone: +48 694101 175, e-mail: mpietrusinska@interia.pl Received: 11.04.2015, accepted: 11.06.2015. 
el through the miRNA structure complementarity with 3'UTR mRNA regions. miRNAs are responsible for regulation of mRNA expression. One miRNA has been proven to be potentially responsible for the expression of many mRNA, and the expression of a single mRNA may be determined by many miRNAs [4].

Their role as factors involved in the development of pathologic conditions is considered, among others, in the context of malignant tumors, diabetes, and recently also pulmonary diseases. What is then the role of miRNA in the development of respiratory disorders?

Dependence in the expression of some miRNA has been shown at the stage of embryonic development of lung tissue. In animal studies on mice, the effect of several dozen miRNAs, whose increased or decreased expression interferes with normal development of the respiratory epithelium has been demonstrated. For instance, the role of miRNA has been shown in repression of the proliferation of pulmonary tissue in late state development (miR-29a) which pathological and increased expression has in turn been shown in the cells of pulmonary tumors [3].

\section{Aim}

The aim of our study is to assess the expression of microRNA in the blood sera of patients diagnosed with bronchial asthma and chronic obstructive pulmonary disease in comparison with healthy subjects. It is a pilot study aiming to determine which microRNAs demonstrate increased levels of expression in the course of the aforementioned pathologies.

\section{Material and methods}

At the initial stage of our research, we investigated the differences in expression of the aforementioned miRNAs in patients with bronchial asthma and chronic obstructive pulmonary disease versus the control group consisting of healthy subjects. First, the groups consisting of 3 patients each were selected. We excluded the patients with concurrent interstitial diseases of the lungs, tumors, end-stage heart failure, and systemic connective tissue diseases. On comparison of the results, such factors as the severity and the level of control of the disease, exacerbations, infections, treatment received by the patients at that time and nicotinism were taken into consideration.

The group of bronchial asthma patients was composed of patients with well-controlled disease (according to GINA 2014 criteria), nonsmokers, without the history of exacerbation periods within the month preceding the study, and using inhaled medications (corticosteroid or corticosteroids and long acting $\beta$-agonist).

The other patient group comprised patients with chronic obstructive pulmonary disease. That group was inhomogeneous with respect to control of the disease one of the patients presented with good control of COPD, treated chronically with inhaled drugs only, with satisfactory spirometric values of forced expiratory volume in $1 \mathrm{~s}\left(\mathrm{FEV}_{1}\right)>60 \%$ of the normal value. The other patients were examined during the period of infectious exacerbations of COPD, while on wide-spectrum antibiotic therapy, systemic steroid therapy with methylprednisolone, or oxygen therapy. It was impossible to perform spirometry in such patients due to the severity of the disease. One female patient is receiving chronic oxygen therapy at home. All the patients were ex-smokers after a minimum 1-year non-smoking period. Due to the fact that this group was inhomogeneous, we could see if there is a difference in microRNA expression in the acute inflammatory process. Healthy subjects, without any chronic diseases, receiving no long-term medications, without the signs of infection within 4 weeks before the study were selected for the control group.

Whole blood samples of $10 \mathrm{ml}$ were collected onto sodium citrate from COPD and asthma patients as well as from healthy subjects. The blood samples were centrifuged at $2.5 \mathrm{~K} \mathrm{RPM}$ to separate plasma, which was then stored at $-20^{\circ} \mathrm{C}$. Total RNA was isolated from the frozen plasma with the use of miRNeasy ${ }^{\circledR}$ Mini Kit (50) (Qiagen) and TRI Reagent ${ }^{\circledR}$ (Sigma Life Science) according to the instructions provided by the manufacturers. The level of RNA was determined using a Picodrop and its quality using a 2100 Bioanalyzer (Agilent Technologies). The isolated RNA samples were stored at $-80^{\circ} \mathrm{C}$. Then reverse transcription reactions were carried out using One Step RT-PCR Kit (25) (Qiagen) and MegaplexTM RT Primers (10X) (Applied Biosystems) according to the manufacturers' recommendations. Isolated RNA in the amount of $3 \mu \mathrm{l}$ was added to the tube to obtain the final reagent volume of $7.5 \mu \mathrm{l}$. The reagent was then incubated $\left(30 \mathrm{~min}, 16^{\circ} \mathrm{C}\right.$ and $30 \mathrm{~min}, 42^{\circ} \mathrm{C}$ ) in a Thermocycler (Biometra). Reverse transcriptase was inhibited $\left(5 \mathrm{~min}, 85^{\circ} \mathrm{C}\right)$. The samples of isolated cDNA were stored at $-20^{\circ} \mathrm{C}$. Subsequently, the pre-amplification reaction was conducted using TaqMan ${ }^{\circledR}$ PreAmp Master Mix (2X) (Applied Biosystems) and MegaplexTM PreAmp Primers (10X) (Applied Biosystems) according to the manufacturers' recommendations. $2.5 \mu \mathrm{l}$ of the product after reverse transcription reaction and $22.5 \mu \mathrm{l}$ of the reaction mix was used for the reaction. The samples were incubated in a Thermocycler (Biometra) (15 s, $95^{\circ} \mathrm{C}$ and $\left.4 \mathrm{~min}, 60^{\circ} \mathrm{C}\right)$. EB buffer in the amount of $75 \mu \mathrm{l}$ was added for dilution. The product was stored at $-20^{\circ} \mathrm{C}$. Then QPCR reaction was carried out using TaqMan Human MicroRNA Array A plates (Applied Biosystems), and a 7900HT Fast Real-Time PCR System cemara (Applied Biosystems). The plates were inoculated with $90 \mu$ l of the product obtained after pre-amplification and incubated in the apparatus for ca. $2 \mathrm{~h}$. All samples were amplified in 3 replicates per plate. The quantitative ratio of miRNAs was analyzed using the comparative Ct method. The quantita- 
tive ratio of miRNAs was analyzed using the comparative Ct method. The amount of miRNA of the studied gene was given as the quantity normalized with respect to the reference gene hsa-miR-39 and was calculated as $2^{-\Delta c t}$. In contrast, the comparative analysis of expression of the gene of interest was presented as the $n$-fold change in comparison with the control in gene expression normalized to the reference gene and was calculated as $2^{-\Delta \Delta c_{t}}$.

\section{Results}

Figure 1 presents the levels of $2^{-\Delta \Delta c_{t}}$ in COPD patients. The analyses demonstrated the most significant differences in the expression of hsa-miRNA-224, hsa-miRNA339-5p, hsa-miRNA-382.

Figure 2 presents the levels of $2^{-\Delta \Delta c_{t}}$ in bronchial asthma patients. The analyses demonstrated the most sig- nificant differences in the expression of hsa-miRNA-224, hsa-miRNA-339-5p, hsa-miRNA-382.

The difference in the amount of miRNA in the plasma between COPD and asthma patients is also noteworthy. In asthma, the expression of hsa-miRNA-224, hsa-miRNA339-5p, hsa-miRNA-382 was 5-fold higher with respect to each of the above miRNAs than in COPD (Figure 3).

\section{Discussion}

The development of research on miRNA in respiratory tract diseases concerns a wide range of both disease entities and materials used for the measurements of miRNA expression. However, there are still vast areas that are not fully understood. Among the diseases affecting the respiratory tract, tumors have been described most extensively with respect to miRNA. Studies of

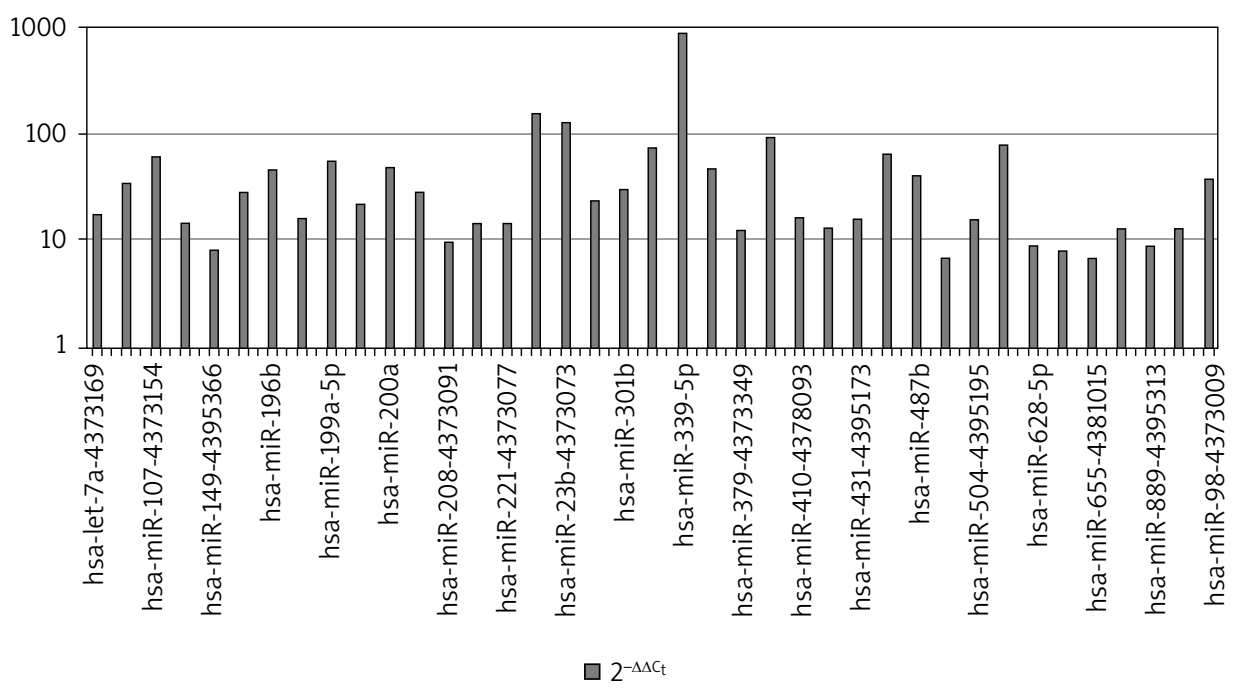

Figure 1. MicroRNA expression in plasma in the group of patients with COPD

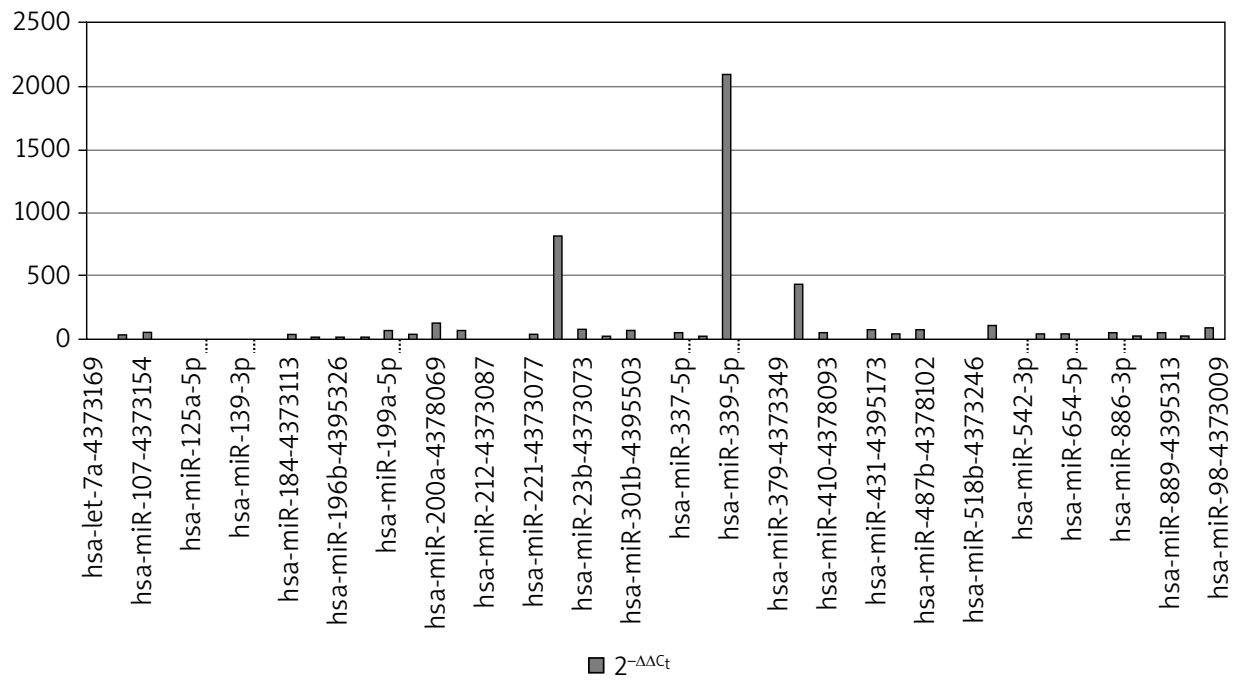

Figure 2. MicroRNA expression in group of patients with asthma 
miRNA in patients with asthma or COPD are characterized by a considerable variety of both the research material and composition of the study group. miRNA was investigated in induced sputum supernatant [3], whole induced sputum [5, 6], blood serum [7-9] as well as saliva [10] or alveolar fibroblasts [11, 12], alveolar macrophages [8]. The search for miRNAs as new biomarkers is conducted very frequently on animal models such as mice or rats $[13,14]$. The spectrum of interest within the area of pulmonary diseases among the studies carried out to date has also been considerably differentiated, including not only obstructive diseases, addressed in our research, but also malignant tumors of the respiratory tract and, less frequently, idiopathic pulmonary fibrosis or cystic fibrosis [3]. Publications concerning the role of miRNAs in the development of pulmonary tissues as early as at the embryonic stage have appeared as well $[3,15]$.

Izzotti et al. demonstrated a two-fold decrease in the expression of 126 miRNAs and a three-fold decrease in the expression of 24 out of 484 miRNAs investigated in the material obtained from rats exposed to tobacco smoke. Among the results of their study, the most significant changes were demonstrated for such miRNAs as let-7, miR-10, miR-26, miR-30, miR-34, miR-99, miR-122, miR-123, miR-124, miR-125, miR-140, miR-145, miR146, miR-191, miR-192, miR-219, miR-222, and miR-22 family, regulating gene expression, responses to stress, apoptosis, angiogenesis and proliferation. Izzotti et al. compared also miRNA expression in the material coming from mice exposed to tobacco smoke. The results were classified not only according to the exposure to pollutants but also according to the age and sex of the animals $[13,14]$

In contrast, the study by Graff et al. investigated the expression of miRNA in alveolar macrophages in smoking vs. non-smoking patients. In that study, decreased global miRNA expression was demonstrated in alveolar macrophages of tobacco smokers in comparison with non-smoking control subjects [12].

The studies on human material collected from healthy subjects and patients with non-small cell lung carcinoma (NSCLC) such as sputum, conducted by Xie et al., demonstrated upregulation of mir-21 in the tumor patients as compared with the healthy controls [6].

Sessa et al. mention a few studies concerning miRNA expression in humans with lung diseases: asthma, COPD, idiopathic pulmonary fibrosis, cystic fibrosis. As far as COPD is concerned, the study by Ezzie et al. attracted their attention. In that study, increased expression of the miR10/107 family, including a significant elevation of miR-15b, the increased expression of which is associated with the presence of emphysema, was observed [3, 16]. Graff et al. found in their study a decrease in the expression of miR-452, the effect of which is higher expression of metalloproteinases (MMP12), correlated with the development of emphysema [3, 12].

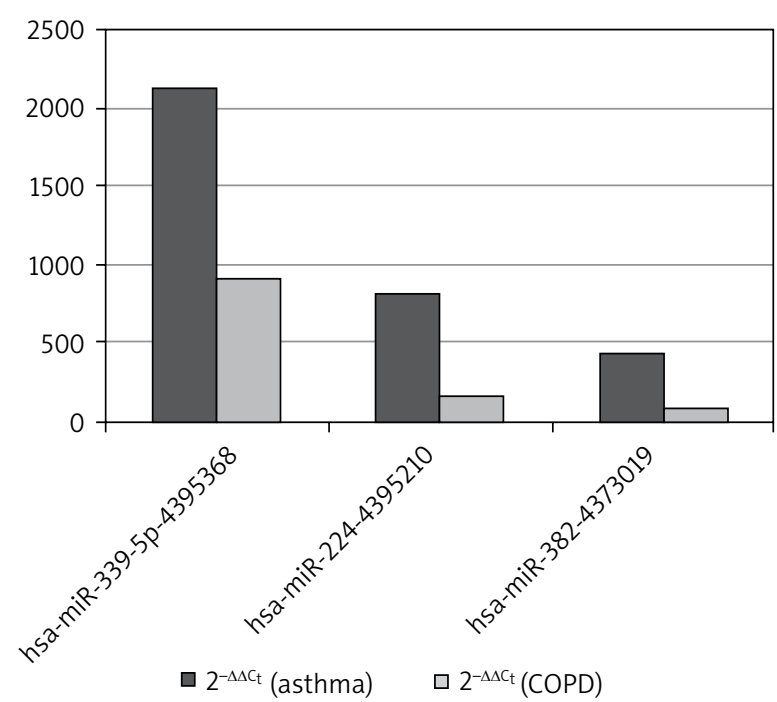

Figure 3. The comparison of three microRNAs, which have shown the highest expression between asthma and COPD

The study by Panganiban et al. assessed the expression of miRNA in the serum of patients with bronchial asthma vs. healthy controls. The control group consisted of patients with the history of allergy (with positive skin tests adopted as a criterion). In $80 \%$ of the asthma patients, an increased expression of miR-1248 and decreased expression of miR-26a, Let7a, and Let7d was demonstrated. A further stage of the study focused on the effect of miR-1248 on the elevated expression of IL-5 - one of the most important cytokines affecting the development of the inflammatory condition in asthma. A positive correlation (an increased miR-1248 expression associated with an increased IL-5 expression) was demonstrated [9].

Gilad et al. analyzed miRNA expression in the sera of pregnant women to find out whether miRNAs are present in systemic fluids (serum, urine, saliva) containing no cellular component and to assess their stability as biomarkers [8].

Akbas et al. investigated the expression of 72 miRNAs in the serum and obtained a result indicating altered regulation of the expression of 5 miRNAs (decreased expression of miR-20a, miR-28-3p, miR-34c-5p, and miR-100 and increased that of miR-7) [7].

Among the miRNA studies, varied and differentiated with respect to the material and group selection, our serum-based study is one of the few belonging to that category. Studies conducted in the groups of asthma or COPD patients are also relatively infrequent. The researchers are more interested in the expression of miRNAs in patients with respiratory tract tumors or those suffering from allergic diseases.

The results of our study differ significantly from those obtained by other authors. Such a discrepancy may result from the difference in the studied material, but also from 
the applied research methodology. It may be also due to the fact that the main interest is focused not on the problem of altered expression in asthma or COPD patients, but on the differences in the expression in active smokers versus subjects who have never smoked, not necessarily presenting any pathologies developed as a result of nicotine addiction.

In our research we intended to compare the expression of miRNAs not only between the healthy controls and the group of patients diagnosed with obstructive diseases, but also between the two disease entities asthma and COPD, which both have similar courses and are underlied by inflammatory processes. The overlap syndrome between them has been identified, which may suggest similar mechanisms and symptoms in the course of both diseases. As demonstrated by our study, the altered expression of miRNA, both in asthma and COPD, involves the same miRNAs, despite the differences in magnitude of the alterations, which may confirm the pathogenetic similarity of both entities.

An increased microRNA expression in plasma may be a potential marker of severity of obstructive pulmonary diseases. The role of microRNA may be found in determining the phenotype of asthma or COPD, what of course has an influence on planning the treatment.

\section{Conclusions}

In view of the results obtained in the preliminary study, we expect to increase the size of the study groups (both patients and controls) and differentiation of the subgroups according to severity, type of treatment, additional factors affecting the course of the disease (e.g. tobacco smoking) within the asthma and COPD patient groups.

\section{Conflict of interest}

The authors declare no conflict of interest.

\section{References}

1. Plank M, Maltby S, Mattes J, Foster PS. Targeting translational control as a novel way to treat inflammatory disease: the emerging role of microRNA. Clin Exp Allergy 2013; 43, 981-99.

2. Booton R, Lindsay M. Emerging role of microRNAs and long noncoding RNAs in respiratory disease. Chest 2013; 146: 193-204.

3. Sessa R, Hata A. Role of micro-RNAs in lung development and pulmonary diseases. Pulm Circ 2013; 3: 315-28.

4. Kupczyk M, Kuna P. MicroRNAs - new biomarkers of respiratory tract diseases. Pneumonol Alergol Pol 2014; 82: 183-90.

5. Van Pottelberge GR, Mestdagh P, Bracke KR, et al. MicroRNA expression in induced sputum of smokers and patients with chronic obstructive pulmonary disease. Am J Respir Crit Care Med 2011; 183: 898-906.
6. Xie Y, Todd NW, Liu Z, et al. Altered miRNA expression in sputum for diagnosis of non-small cell lung cancer. Lung Cancer 2010; 67: 170-6.

7. Akbas F, Coskunpinar E, Aynaci E, et al. Analysis of serum micro-RNAs as potential biomarker in chronic obstructive pulmonary disease. Exp Lung Res 2012; 38: 286-94.

8. Gilad S, Meiri E, Yogev Y, et al. Serum microRNAs are promising novel biomarkers. PLoS One 2008; 3: e3148.

9. Panganiban RP, Pinkerton M, Maru S, et al. Differential microRNA expression in asthma and the role of miR-1248 in regulation of IL-5. Am J Clin Exp Immunol 2012; 1: 154-65.

10. Park NJ, Zhou H, Elashoff D, et al. Salivary microRNA: discovery, characterization, and clinical utility for oral cancer detection. Clin Cancer Res 2009; 15: 5473-7.

11. Sato T, Liu X, Nelson A, et al. Reduced miR-146a increases prostaglandin $\mathrm{E}$ in chronic obstructive pulmonary disease fibroblasts. Am J Respir Crit Care Med 2010; 182: 1020-9.

12. Graff J, Powers L, Dickson A, et al. Cigarette smoking decreases global microRNA expression in macrophages. PLoS One 2012; 7: e44066.

13. Izzotti A, Calin GA, Arrigo P, et al. Downregulation of microRNA expression in the lungs of rats exposed to cigarette smoke. FASEB J 2009; 23: 806-12.

14. Izzotti A, Calin GA, Steele VE, et al. Relationships of microRNA expression in mouse lung with age and exposure to cigarette smoke and light. FASEB I 2009; 23: 3243-50.

15. Harris KS, Zhang Z, McManus MT, et al. Dicer function is essential for lung epithelium morphogenesis. Proc Natl Acad Sci USA 2006; 103: 2208-13.

16. Ezzie ME, Crawford M, Cho JH, et al. Gene expression networks in COPD: microRNA and mRNA regulation. Thorax 2012; 67: 122-31. 